\title{
Exercise Practice Among Women Attending Antenatal Care at the University Teaching Hospital in Lusaka, Zambia
}

\author{
Loveness A. Nkhata ${ }^{*}$, Esther Munalula-Nkandu, Hastings Shula \\ Department of Physiotherapy, School of Medicine, University of Zambia, Lusaka, Zambia
}

Email address:

Lnkhata@yahoo.com (L. A. Nkhata)

To cite this article:

Loveness A. Nkhata, Esther Munalula-Nkandu, Hastings Shula. Exercise Practice Among Women Attending Antenatal Care at the University Teaching Hospital in Lusaka, Zambia. Science Journal of Public Health. Vol. 3, No. 3, 2015, pp. 361-365.

doi: $10.11648 /$ j.sjph.20150303.19

\begin{abstract}
Introduction: In the absence of medical or obstetrical complications, pregnant women are encouraged to continue and maintain active lifestyles during their pregnancies. Benefits of exercise during pregnancy include; prevention of excessive gestational weight gain, gestational diabetes, pre-eclampsia, undergoing a caesarean section and reduced low back pain. The aim of the study was to obtain information on exercise practice among women attending antenatal care at the University Teaching Hospital (UTH) in Lusaka, Zambia and establish whether, educational level, number of pregnancies and cultural background had an influence on exercise practice during pregnancy. Methods: We collected data using a self- administered questionnaire in a cross sectional, exploratory study and summarized data using descriptive statistics. The chi-square test was used to test association between variables and the significance level was set at 5\%. Results: Three hundred women participated in the survey. The majority of the respondents $74 \%(n=222)$ exhibited inadequate levels of knowledge on exercise and the type of ideal exercises. Exercise practice in relation to the educational levels was insignificant. However, cultural background had a significant association to the women's exercise practice (p-value 0.025 ). In addition, the number of pregnancies was positively associated to the women's exercise practice (p-value 0.01) during pregnancy. Conclusions: Pregnant women practice general physical activities of daily living such as walking and household chores during pregnancy. They do not know the specific antenatal exercises. Consequently, they are not able to practice the ideal exercise during pregnancy. This highlights the need for Physiotherapy personnel to be actively involved during antenatal to educate pregnant women on the ideal exercise activities.
\end{abstract}

Keywords: Antenatal, Exercise, Knowledge, Practice, Pregnancy, Physiotherapy

\section{Introduction}

In the absence of medical or obstetrical complications, pregnant women are encouraged to continue and maintain active lifestyles during their pregnancies [1, 2, 3, 4, 5, 6,7]. In addition, exercise has many benefits including reducing the risk for coronary heart disease, cardiovascular disease, metabolic syndrome and systemic inflammation. In pregnancy, appropriate exercise decreases the risk of developing gestational diabetes mellitus, pre-eclampsia, caesarean section rate, reduced symptoms from low back pain and improved mental state [2, 3, 5, 6, 7]. Furthermore, babies born from exercising women seem calmer, more intelligent with improved neurological and mental development and adapt faster to the outside. However, according to Piravej and Sakisirinukul [8] most pregnant women are less active during pregnancy; the rates of activity differ by race and ethnicity in a number of ways [7, 8, 9]. Personal and cultural values are believed to have an influence on the women's physical activity behaviors and little is known about how to support exercises during this period. Motolla and McLaughlin [1] suggest that over time, the attitude towards pregnancy has changed. Going from beliefs that women should not stress their bodies unduly to accepting that exercise could positively affect the health of the mother and child is part of that change [2, 3, 4]. Nonetheless, studies have reported that many expectant mothers still remain inactive and do not meet sufficient exercise recommendations $[5,6,7,8,9,10]$. The major reason is potential adverse effects on themselves and their unborn child $[9,10]$.

Safe motherhood educational programs in Zambia are done during antenatal clinics. Pregnant women are taught 
about prevention of mother to child transmission (PMTCT), good nutritional habits and ground immunisations to mention a few. They are also encouraged to exercise to ensure a healthy birth. However, there is inadequate information on exercise practice among women attending antenatal care in Zambia. Therefore, we conducted a survey to explore exercise practice among women attending antenatal care at the UTH and establish whether, educational level, number of pregnancies and cultural background had an influence on exercise practice during pregnancy.

\section{Methods}

We recruited 300 pregnant women in a cross-sectional survey. The sample size was large enough to mitigate the possibility of variability in the response variables. Ethical approval was obtained from the Biomedical Research Ethics Committee of the University of Zambia (UNZABREC). Data was collected using a self-administered questionnaire, which was divided, into three parts. Demographic data was collected in section A while data on antenatal care and previous pregnancies was collected under section $\mathrm{B}$ and in Section $\mathrm{C}$ exercise practice in pregnancy. Further, this section set out to determine the willingness of women to seek medical advice on exercise practice in current and previous pregnancies, type of exercises done and frequency. Analysis of data was done using the statistical package for social sciences (SPSS) version 17.0 for windows and summarized using descriptive statistics. Association between variables was tested using the chi-square test and significance level was set at $5 \%$.

\section{Results}

Three hundred pregnant women participated in the survey; the mean age was 29.4 years. Most participants $48 \%(n=144)$ had attained college level of education and $44 \%(n=132)$ were in formal employment (Table 1).

Table 1. Respondents Demographic characteristics.

\begin{tabular}{lll}
\hline Age & Frequency & Percentage \\
\hline $20-25$ & 33 & $11 \%$ \\
$26-30$ & 134 & $45 \%$ \\
$31-35$ & 100 & $33 \%$ \\
$36-40$ & 33 & $11 \%$ \\
Educational Background & & \\
Primary & 24 & $8 \%$ \\
Secondary & 132 & $44 \%$ \\
College & 144 & $48 \%$ \\
Occupational Background & & \\
Student & 12 & $4 \%$ \\
Unemployed & 66 & $22 \%$ \\
Formal employment & 132 & $44 \%$ \\
Self employed & 90 & $30 \%$ \\
\hline
\end{tabular}

\subsection{Antenatal Care Attendance}

Majority of the pregnant women $96 \%(n=288)$ attended antenatal care regulary, $74 \% \quad(n=222)$ attached great importance to antenatal attendance while the average number of pregnancies reported was three (Table 2).

Table 2. Antenatal attendance, reported exercise activities and source of information.

\begin{tabular}{lll}
\hline Antenatal Attendance & Frequency & Percentage \\
\hline Yes & 288 & $96 \%$ \\
No & 12 & $4 \%$ \\
Exercise during pregnancy & & \\
Yes & 207 & $69 \%$ \\
No & 93 & $31 \%$ \\
Reported exercise activities & & \\
Walking & 87 & $29 \%$ \\
Walking and sit ups & 33 & $11 \%$ \\
Walking and sweeping & 39 & $13 \%$ \\
Breathing exercises and sit ups & 42 & $14 \%$ \\
Sit ups and Leg raising & 33 & $11 \%$ \\
Leg raising & 18 & $6 \%$ \\
Washing and sweeping & 33 & $11 \%$ \\
Sweeping & 15 & $5 \%$ \\
Sources of information & & \\
Television & 105 & $35 \%$ \\
Nurses & 75 & $25 \%$ \\
Internet and books & 60 & $20 \%$ \\
Doctors & 45 & $15 \%$ \\
Physiotherapists & 15 & $5 \%$ \\
\hline & & \\
\hline
\end{tabular}

\subsection{Respondents' Exercise Practice During Pregnancy,} Type of Exercise Activity and Sources of Information

Only 69\% $(n=207)$ reported practicing some form of exercise during the current pregnancy and 63\% $(n=189)$ in previous pregnancies. Walking $29 \%(n=87)$ was the most common type of exercise identified by respondents (Table 2). The common source of information for most respondents $35 \%(n=107)$ was television and books. Only 5\% reported having obtained information from a physiotherapist.

\subsection{Seeking Medical Advice During Pregnancy}

Most participants 93\% ( $\mathrm{n}=279)$ identified with seeking medical advice on exercise during pregnancy (Table 2). Reasons for seeking medical advice included knowing the right exercise to do $56 \%(\mathrm{n}=168)$ and proper positions when exercising $19 \%(n=57)$.

\subsection{Cross Tabulations for Educational Level, Number of Pregnancies and Cultural Background to Exercise Practice}

Exercise practice in relation to the respondents' educational level was statistically significant ( $p$-value 0.023 ) while cultural background had a significant association to the women's exercise practice ( $\mathrm{p}$-value 0.008). In addition, the number of pregnancies was positively associated to the women's exercise practice ( $\mathrm{p}$-value $\leq 0.001$ ) during 
pregnancy (Table 3).

Table 3. Cross tabulations for Educational levels, cultural background and number of pregnancies to exercise practice.

\begin{tabular}{lllll}
\hline Category & Chi $^{-}{ }_{\text {statistic }}$ & p-value significance level & p-value result & Comment \\
\hline Educational level & 5.1282 & 0.05 & 0.024 & significant \\
Cultural background & 13.6728 & 0.05 & 0.008 & significant \\
Number of pregnancies & 42.053 & 0.05 & $\leq 0.001$ & significant \\
\hline
\end{tabular}

\section{Discussion}

While, exercise is a fundamental component of antenatal care, women's physical activity lessens, as the perception of risk during pregnancy is high [11]. It is recommended that women should continue exercising during pregnancy as in most cases; exercise is safe for both mother and foetus $[1,2$, $3,7]$. In addition, benefits of exercise in pregnancy include improved physical fitness, cardiovascular endurance, and prevention of excessive gestational weight gain and glucose intolerance. Most of the pregnant women that participated in the study had attained formal education and attached great importance to antenatal attendance. Exercise practice was significantly associated to the level of education amongst the pregnant women that participated in this study. This result is different from what was reported in Brazil [12] where no statistically significant association was found between the practice of exercise and education level or being in paid employment. Though, level of education was statistically significant the majority of the women exhibited inadequate levels of knowledge on ideal exercises during pregnancy. Their information on exercise during pregnancy lacked detail and was inconsistent with little specificity. In contrast, Riberio and Milanez [12] in their study reported that most women were adequately informed about the practice of physical exercise during pregnancy and significant associations were found between adequate knowledge of physical exercise during pregnancy and their education level. A number of factors were identified as contributing to pregnant women's inability to meet exercise recommendations. These include but are not limited to; safety concerns, previous involvement in regular exercise, level of knowledge, level of education, personal attitudes, beliefs and phobia among pregnant women $[9,10,11]$. In this study, the majority of the women reported not knowing how to perform antenatal exercises, feeling tired and uncomfortable as reasons for not doing exercises. Similar results were submitted in Nigeria [13] and Brazil [12]. Therefore, emphasis on exercise education should be a priority in antenatal care to adequately inform women on exercise practice and benefits during pregnancy. To encourage proper exercise in pregnancy, Piravej \& Sakisirinukul [8] recommend that all members of the related health care team should be involved in planning. Physiotherapists should be integrated in the antenatal and reproductive health care programmes at UTH to provide information and training on exercise activities ideal for pregnancy, because they are professionals in this area and can make meaningful contributions in reproductive health care provision.
Often, it is assumed that multiparous women are able to handle subsequent pregnancies better than the nulliparous because of their experiences and knowledge gained from earlier pregnancies. The average number of pregnancies reported in this study was three. History of the respondent's previous pregnancies was noted and enquiry made into whether mothers exercised during previous pregnancies. Outcomes revealed that there was a positive association between the number of pregnancies and the women's exercise practice during pregnancy. However, the women's knowledge on the ideal antenatal exercises done during pregnancy was limited. This may suggest that advice received from health care personnel during antenatal clinics on the types of exercises done during pregnancy is inadequate as most of the women reported having attended antenatal clinics even in previous pregnancies. At least 30 7 minutes of moderate non-weight bearing exercises a day is recommended during pregnancy $[1,2,4]$. In addition, the safest and most productive activities during pregnancy include; swimming, brisk walking, indoor stationary bicycling and low-impact aerobics. These activities carry little risk of injury, benefit the entire body, and can be continued until birth. Furthermore, activities such as jogging, tennis and racquetball are generally safe but must be done in moderation because changes in balance during pregnancy may affect rapid movements. Walking was the most common type of exercise identified by respondents. This is similar to the outcomes reported by Riberio and Milanez [12] and may be attributed to the fact that the walking activity is easy to carry out and there are no costs or equipment involved when performing the activity.

Culture is a way of life for a group of people and a full range of learned human behaviour patterns [5, 14]. It plays a major role in patient compliance and differences in culture affect patients' attitudes towards medical care and their ability to understand, manage, and cope with the consequences of medical treatment [15]. According to McLaughlin and Braun [5] all cultures have systems of health beliefs to explain what causes illness, how it can be cured or treated, and who should be involved in the process. In addition, different groups have their own perspectives and values to the health care system, and many health care beliefs and health practices differ from the traditional health care culture [15]. Furthermore, the extent to which patients perceive patient education as having cultural relevance for them can have a profound effect on their reception to information provided and their willingness to use it. Participants who took part in this study belonged to five subcultures here in Zambia. Outcomes revealed that cultural background had a significant association to the women's 
exercise practice. In comparison to Mbada, et al [13] social demographic characteristics may have influenced the outcomes. This is because participants dwell in the city, which is characterised by a combination of cultures therefore, others factors may have interacted and contributed to the women's exercise practice during pregnancy.

Substantial amount of research has been completed to support the idea that it is beneficial to exercise during pregnancy. Progress from 15 to 30 minutes of continuous aerobic exercises at least 3 to 4 times per week for pregnant women with no contraindications is recommended [1, 2, 4].At UTH, antenatal health talks have a focus on HIV/AIDS, prevention of mother to child transmission, malaria prevention, nutrition, ground immunisations and mother's general fitness. Exercise activities are encouraged among pregnant women but details on how to perform specific antenatal exercises and how to perform them during pregnancy is inadequate. Therefore, pregnant women look for information from a wide range of sources. These include magazines, media, friends, doctors, nurses, and rarely, physiotherapists. Regardless of previous pregnancy, outcomes and experiences participants who took part in this study were willing to engage in physical activity during pregnancy. This is evident in the number of women who reported doing brisk walking and participating in various household chores as a form of physical activity during pregnancy. This study recommends that exercise practice during pregnancy should be emphasised but be prescribed. Physiotherapy personnel being the custodians and professionals inclined to physical activity must be actively involved to educate and advise pregnant women on antenatal exercises they should engage in. These include; deep breathing exercises, foot and ankle exercises, pelvic floor muscle exercises, abdominal exercises and relaxation [5].

Outcomes from this study cannot be extrapolated to the female population even though they may face similar situations during pregnancy. This is because information at the time of data collection was obtained only among pregnant women that attended antenatal clinics at the UTH and is based on individual responses. There is also a possibility of instrument bias because the questionnaire employed in the study may not have been calibrated adequately.

\section{Conclusion}

Pregnant women practice general physical activities of daily living such as walking and household chores during pregnancy. Nonetheless, pregnant women may not know the specific antenatal exercises. Consequently, they may not able to practise the ideal exercise during pregnancy. Therefore, Physiotherapy personnel must be actively involved during antenatal to educate pregnant women on the exercise activities.

\section{Acknowledgements}

This work arose from LAN's dissertation that was submitted to the University of Zambia (UNZA) as partial fulfillment for the award of the Bachelor of Science degree in Physiotherapy in the Department of Physiotherapy of the School of Medicine. We thank management of the University Teaching Hospital and members of the Department of Physiotherapy of UNZA for having contributed in one way or another to the work.

\section{References}

[1] Mottola, M.F, and McLaughlin, R. (2011) Exercise and Pregnancy: Canadian Guidelines for Health Care Professionals. Wellspring, 22(4), A1-A4.

[2] American Congress of Obstetricians and Gynaecologists (ACOG) and Committee on Obstetric Practice, (2002) "Exercise during pregnancy and the postpartum period'. ACOG Committee Opinion Number 267," Obstetrics \& Gynaecology, 99(1), 171-173.

[3] Clark A.M (2005) Promoting exercise during pregnancy: A qualitative study of pregnant women, birth partners and health professionals in Scotland. University of Glasgow.

[4] Zavorsky G. S\& Longo, L. D. (2011).Exercise Guidelines in Pregnancy. Sports Medicine, 41(5), 345-360.

[5] McLaughlin L and Braun K. (1998) "Asian and Pacific Islander cultural values: Considerations for health care decision-making." Health and Social Work, 23 (2), 116-126.

[6] Piravej K and Sakisirinukul R (2001) Survey of exercise patterns, attitudes and the general effects of exercise during pregnancy in 203 Thai Women at King Chulalongkorn Memorial Hospital, Thailand. www.pubmed.gov

[7] Paisley T.S, Joy E.A and Price R.J (2003) Exercise during pregnancy a practical approach. www.pubmed.gov

[8] Ribeiro C.P and Milanez H (2011)Knowledge, attitude and practice of women in Campinas, S ao Paulo, Brazil with respect to physical exercise in pregnancy: a descriptive study. Reproductive Health, 8(1), 1-7

[9] Mbada C.E, Adebayo O.E, Adeyemi A.E, Arije O.O, Dada O.O, Akinwande O.A, Awotidebe, T.O and Alonge I.O (2014) Knowledge and Attitude of Nigerian Pregnant Women towards Antenatal Exercise: A Cross-Sectional Survey. ISRN Obstetrics and Gynecology, Article ID 260539, 1-8

[10] Weallens E, Clark A.M, Mutrie N and Gaudoin M (2003) Attitudes and beliefs of Primegravidae women to exercise during pregnancy. Health Educational Journal, Glasgow, Scotland.

[11] Evenson K.R, Moos M, Carrier K, and Siega-Riz A. M, (2009) Perceived barriers to physical activity among pregnant women. Maternal and Child Health Journal, 13(3), 364-375.

[12] Duncombe D, Wertheim E. H, Skouteris H, Paxton S. J, and Kelly L, (2009) Factors related to exercise over the course of pregnancy including women's beliefs about the safety of exercise during pregnancy. Midwifery, 25(4), 430-438.

[13] Wang T.W and Apgar B.S (2000) Exercise during pregnancy. American Family Physician, 57(8), 1846-1852. 
[14] Clapp J.F (2000) Beginning regular exercise in early pregnancy: Effects on fetoplacental growth. American Journal of Obstetrics and Gynaecology 183:6-10
[15] Helman, C.G (1994) Culture, Health and Illness: An Introduction for Health Professionals. ButterworthHeinemann, Oxford, England. 101-145. 